\title{
SUPERFLUID CHARACTERISTICS OF INDUCED-PAIRING MODEL
}

\author{
W.R. Czar'T, M. Szkudlarek and S. Robaszkiewicz \\ Institute of Physics, A. Mickiewicz University \\ Umultowska 85, 61-614 Poznań, Poland
}

We study the electromagnetic and thermodynamic properties of a model of coexisting local electron pairs and itinerant carriers coupled via the intersubsystem charge exchange. The calculations of the London penetration depth, the energy gap, the magnetic critical fields and the coherence length in the superconducting phase are performed. The effects of reduced binding energy of local pairs are discussed.

PACS numbers: 74.20.-z, 71.28. $+\mathrm{d}, 74.25 . \mathrm{Ha}$

A model of a mixture of tightly bound pairs and wide-band electrons was introduced several years ago [1] for a unified description of nonconventional superconductors, chalcogenide glasses, nonsimple metals and the systems with alternate valency. It has been extensively analysed in several more recent papers (see Refs. $[2,3]$ and papers quoted therein). Here we discuss the superfluid characteristics of such a system taking as a starting point a generalization of the original model, proposed in Ref. [4].

The considered effective Hamiltonian of coexisting localized $d$-electrons and itinerant $c$-electrons can be written as

$$
\begin{aligned}
& H=\sum_{i \sigma}\left(E_{0}-\mu\right) n_{i \sigma}^{d}+U \sum_{i} n_{i \uparrow}^{d} n_{i \downarrow}^{d}+t \sum_{i j \sigma}\left(\mathrm{e}^{\mathrm{i} \Phi_{i j}} c_{i \sigma}^{+} c_{j \sigma}+\text { h.c. }\right)-\sum_{i \sigma} \mu c_{i \sigma}^{+} c_{i \sigma} \\
& +\frac{I_{0}}{2 N} \sum_{i k k^{\prime}}\left(\mathrm{e}^{\mathrm{i}\left(k-k^{\prime}\right) R_{i}} c_{k \uparrow}^{+} c_{k^{\prime} \downarrow}^{+} \rho_{i}^{-}+\text {h.c. }\right) \quad\left(n_{i \sigma}^{d}=d_{i \sigma}^{+} d_{i \sigma}, \rho_{i}^{-}=d_{i \downarrow} d_{i \uparrow}\right),
\end{aligned}
$$

where $E_{0}$ measures the relative position of $d$-level with respect to the bottom of the $c$-electron band $\epsilon_{k}$ in the absence of interactions, $\mu$ is the chemical potential which ensures that a total number of particles is constant, i.e. $n=n_{c}+n_{d}=$ $\left(\sum_{k \sigma}\left\langle c_{k \sigma}^{+} c_{k \sigma}\right\rangle+\sum_{i \sigma}\left\langle n_{i \sigma}^{d}\right\rangle\right) / N, U$ is the effective on-site density interaction between $d$-electrons, $t$ is the hopping integral for $c$-electrons and $I_{0}$ is the intersubsystem charge exchange coupling. The Peierls factor in Eq. (1) account for the coupling of electrons to the magnetic field via its vector potential $\boldsymbol{A}(\boldsymbol{r})$. $\Phi_{i j}=-\frac{e}{\hbar c} \int_{R_{i}}^{R_{j}} \mathrm{~d} \boldsymbol{r} \boldsymbol{A}(\boldsymbol{r})$, and $e$ is the electron charge.

In analysis we used the variational approach which treats the on-site interaction term $U$ exactly $[4,5]$ and the intersubsystem interaction within the broken 
symmetry HFA [1]. For $A=0$ the resulting expression for the free energy of the superconducting phase $(S)$ is

$$
\begin{aligned}
\frac{F_{0} \mathrm{~S}}{N} & =-\frac{2}{\beta N} \sum_{k} \ln \left(2 \cosh \frac{\beta A_{k}}{2}\right)-\frac{1}{\beta} \ln 2 Z_{d}+\mu\left(n_{d}+n_{c}\right) \\
& -\bar{\mu}-\mu+2\left|I_{0}\right| x_{0} \rho_{0},
\end{aligned}
$$

where $A_{k}=\left[\left(\epsilon_{k}-\mu\right)^{2}+\left(I_{0} \rho_{0}\right)^{2}\right]^{1 / 2}, \quad Z_{d}=\exp (\beta U / 2)+\cosh \beta \Delta, \Delta=$ $\sqrt{\bar{\mu}^{2}+\left(I_{0} x_{0}\right)^{2}}, \bar{\mu}=\mu-E_{0}-U / 2, \beta=\left(k_{\mathrm{B}} T\right)^{-1}, \rho_{0}=(1 / N) \sum\left\langle\rho_{i}^{+}\right\rangle$and $x_{0}=(1 / N) \sum_{k}\left\langle c_{k}^{+} c_{-k \downarrow}^{+}\right\rangle$, are the order parameters for $d$ and $c$ subsystems, respectively, and $E_{\mathrm{g}}(T)=\min 2 A_{k}$ is the energy gap in the $c$-electron spectrum. The optimum values of $\mu, x_{0}$ and $\rho_{0}$ are determined by the set of equations $\frac{\partial F_{0} \mathrm{~s}}{\partial \mu}=0, \frac{\partial F_{0} \mathrm{~s}}{\partial x_{0}}=0, \frac{\partial F_{0}^{\mathrm{s}}}{\partial \rho_{0}}=0$, and the free energy of the normal $(\mathrm{N})$ state $F_{0}^{\mathrm{N}}$ is obtained from (2) by putting $x_{0}=\rho_{0}=0$, and with $\mu$ given by $\frac{\partial F_{0}^{N}}{\partial \mu}=0$.

For a weak static potential the expectation value of the Fourier transform of the total current operator can be obtained from the linear response theory as $J_{\alpha}(q)=\sum_{\beta}\left[\delta_{\alpha \beta} K^{\text {dia }}+K_{\alpha \beta}^{\text {para }}(q)\right] A_{\beta}(q)$, and the diamagnetic part of kernel evaluated within our approximation scheme has the form

$$
K^{\mathrm{dia}}=\frac{8 \pi e^{2}|t|}{\hbar^{2} c^{2} a_{\perp}} \frac{1}{N} \sum_{k}\left[1-\frac{\left(\epsilon_{k}-\mu\right)}{A_{k}} \tanh \left(\frac{\beta A_{k}}{2}\right)\right] \cos k_{\alpha} \quad(\alpha=x, y, z) .
$$

In the local approximation the penetration depth may be expressed in terms of the transverse part of the total kernel as $\lambda=1 /\left[-K_{x x}\left(0, q_{y} \rightarrow 0,0\right)\right]^{1 / 2}$, and at $T=0$ it is determined entirely by $K^{\text {dia }}$ because $K_{\alpha \alpha}^{\text {para }}(q)$ vanishes in the $q \rightarrow 0$ limit. Using the value of the penetration depth and the difference of the free energy between $N$ and $S$ phase one is able to determine the thermodynamic critical field $H_{\mathrm{c}}$ and the Ginzburg-Landau correlation length $\xi_{\mathrm{GL}}$ as $H_{\mathrm{c}}{ }^{2}(T) /(8 \pi)=\left[F^{\mathrm{N}}(T)-F^{\mathrm{S}}(T)\right] / N a^{3}, \xi_{\mathrm{GL}}=\Phi_{0} /\left(2 \pi \sqrt{2} \lambda H_{\mathrm{c}}\right)$, where $a$ is a lattice constant, $\Phi_{0}=h c / 2 e$, and to obtain the estimations for the critical fields $H_{\mathrm{c} 1} \approx \frac{\ln \kappa}{\kappa} H_{\mathrm{c}}$ and $H_{\mathrm{c} 2} \approx \Phi_{0} /\left(2 \pi \xi_{\mathrm{GL}}^{2}\right)$, where $\kappa=\lambda / \xi_{\mathrm{GL}}$.

In the model considered superconductivity can only occur provided that both subsystems participate in its formation. Depending on the relative concentration of " $c$ " and " $d$ " electrons which is determined by $\Delta_{0}=E_{0}+U / 2$ and $n$ we distinguish 3 essentially different physical situations: (i) $d$-regime, $n_{d} \gg n_{c}$, (ii) $d+c$ (mixed valence) regime, $2>n_{c}, n_{d}>0$, (iii) $c$-regime, $n_{c} \gg n_{d}$. In $d$-regime the superconducting properties of the systems are analogous (at least for $U \leq 0$ ) to those of pure local-pair superconductor, whereas in $c$-regime the situation is similar to the BCS case: pairs of " $c$ " electrons with opposite momenta and spins are exchanged via virtual transitions into empty $d$-levels. In the most interesting $d+c$ regime the system shows features which are intermediate between those of pure local pair superconductor and those of classical BCS systems, even in the strong attraction $(U \ll 0)$ case. This concerns, among others, the gap in the single-particle excitation spectrum for $c$-electrons, the ratio $k_{\mathrm{B}} T_{\mathrm{c}} / E_{\mathrm{g}}(0)$, the electromagnetic coherence length, the thermodynamic critical field, as well as the properties of the normal state. 

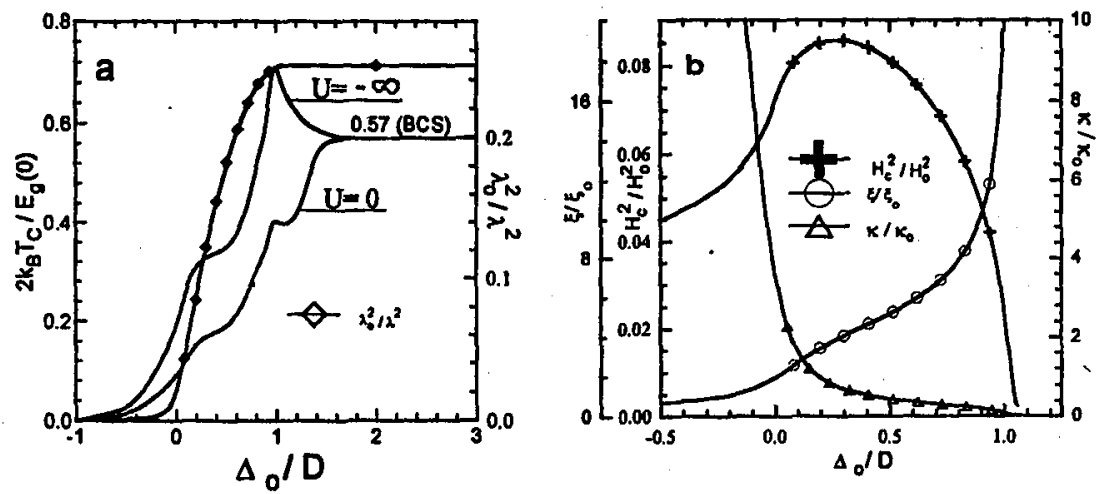

Fig. 1. The plots of $2 k_{\mathrm{B}} T_{\mathrm{c}} / E_{p}(0), 1 / \lambda^{2}$ (a) and $\xi, \kappa, H_{\mathrm{c}}^{2}$ (b) as a function of $\Delta_{0} / D$ for $n=1, U \leq 0, \lambda_{0}^{2}=\hbar^{2} c^{2} a_{\perp} /\left(16 \pi e^{2} D\right), H_{0}^{2}=16 \pi D / a_{\perp} a_{\|}^{2}, \xi_{0}=a_{\|} /(4 \sqrt{2}), \kappa_{0}=$ $\frac{\sqrt{2} h c}{\sqrt{\pi} e} \frac{\sqrt{a_{\|}}}{a_{\|} \sqrt{D}} ; D=Z t, Z$ - number of nearest neighbours, $I_{0} / 2 D=0.1$.
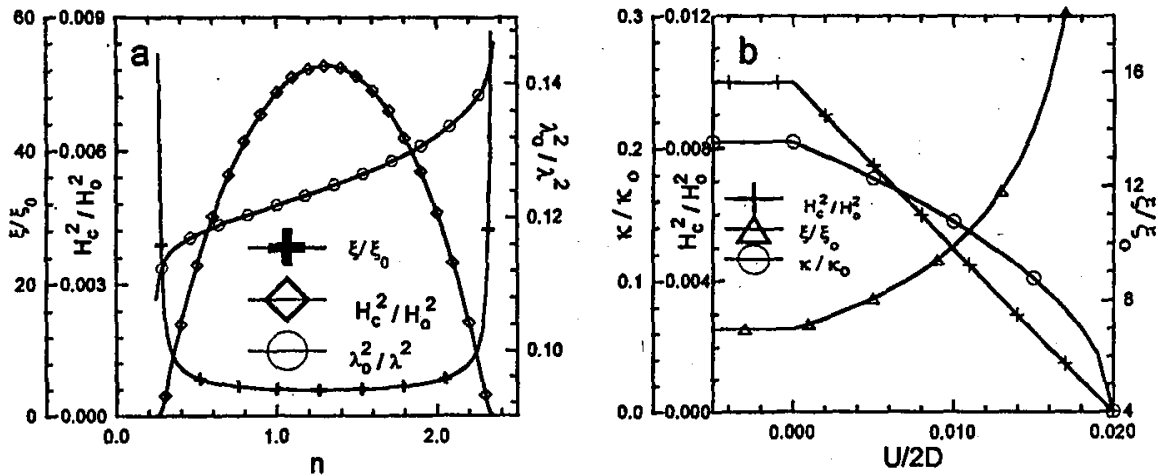

Fig. 2. Concentration dependence of $\xi, 1 / \lambda^{2}$ and $H_{c}^{2}$ for $\Delta_{0}=0.3, U \leq 0$ (a) and the plots of $\xi, 1 / \lambda^{2}$ and $H_{c}^{2}$ vs. $U / 2 D$, for $n=2, \Delta_{0} / D=1$ (b); $I_{0} / 2 D=0.1$.

In Figs. 1 and 2 we present the results of numerical calculations of various superfluid characteristics, obtained using a rectangular density of states for $c$-electrons.

Figure 1a shows the variation of the ratio $2 k_{\mathrm{B}} T_{\mathrm{c}} / E_{\mathrm{g}}(0)$ and $\lambda^{-2}$ as a function of $\Delta_{0} / D$ for $n=1$. As we see, the ratio approaches the BCS value in $c$-regime and it strongly decreases as the concentration of $d$-electrons increases above that of wide-band electrons with decreasing $\Delta_{0}$. The London penetration depth $\lambda(0)$ being very large in the local pair limit $\left(\Delta_{0}<0\right)$ decreases strongly with increasing $\Delta_{0}$ in $d+c$ regime, whereas in $c$-regime it remains almost constant. The crossover between the local-pair-like and BCS-like behaviours is very clearly seen in the variation of the coherence length $\xi$ and the Ginzburg ratio $\kappa$ with increasing $\Delta_{0}$ (Fig. 1b). For $\Delta_{0} \leq 0 \xi_{\mathrm{GL}}(\kappa)$ is very small (large), indicating extreme type II superconductivity, and $\xi_{\mathrm{GL}}(\kappa)$ rapidly increases (decreases) as one approaches the $c$-regime.

Decreasing $|U|(U \leq 0)$ yields a moderate reduction of $T_{c}$ (up to a factor 2 for $U \rightarrow 0$ ), in comparison to the $U=-\infty$ case [4] and the analogous reduction 
of the ratio $k_{\mathrm{B}} T_{\mathrm{c}} / E_{\mathrm{g}}(0)$ in the (i) and (ii) regimes, whereas in the (iii) regime $k_{\mathrm{B}} T_{\mathrm{c}} / E_{\mathrm{g}}(0)$ remains almost unaffected by $U$ and it approaches the BCS value (0.57) for any $U \leq 0$ (see Fig. 1a).

In Fig. 2a we show the evolution of $H_{c}, \lambda$ and $\xi$ with $n=n_{d}+n_{c}$, for a fixed value of $\Delta_{0} / D$ and $U \leq 0$. Upon increasing $n$ the system exhibits a change-over from the $c$-regime $\left(n<n_{1}, n_{d} \approx 0\right)$ into the $c+d$ regime and then again into the $c$-regime $\left(n>n_{2}, n_{d} \approx 2\right)$. Notice only a weak $n$-dependence of $\lambda$ for $n_{1}<n<n_{2}$, which is due to a strong pinning of $\mu$ around the $d$-level $\left(\Delta_{0}\right)$ in this regime.

In the considered model the $S$ state can develop not only for the attractive $U(-\infty \leq U \leq 0)$ but also for the repulsive $U$ (if $0<U<U_{c}$ ) [4]. In general, for weak repulsion the superconducting transition remains continuous, as for $U<0$, however the $d$-pair density exhibits a sharp break at $T_{\mathrm{c}}$ and the ratio $k_{\mathrm{B}} T_{\mathrm{c}} / E_{\mathrm{g}}(0)$ is reduced. The increasing $U$ changes the nature of the phase transitions from a continuous to a discontinuous type, resulting in the tricritical point, and finally, for $U>U_{\mathrm{c}}$ the system remains in the normal state at any temperature.

For repulsive $U$ even in the $d$-regime the properties of the system are in many aspects very different from those of standard local-pair superconductor: $T_{c}$ is determined by the $d$-pair breaking excitation (not by pair mobility) and for a small $d$-pair binding energy (i.e. for substantial values of $U$ ) there are essentially no preformed $d$-pairs above $T_{\mathrm{c}}$. In Fig. $2 \mathrm{~b}$ we present the plots of $H_{\mathrm{c}}^{2}, \xi_{\mathrm{GL}}$ and $\kappa=\lambda / \xi$ as a function of $U / 2 D$, for two fixed values of $I_{0} / 2 D\left(n=2, \Delta_{0} / D=1\right)$.

A more extended discussion of the model for $U>0$ with a Ginzburg-Landau analysis of the electromagnetic and thermodynamic properties of the system in the case of first-order superconducting transitions $\left(U \leq U_{c}\right)$ will be given in a subsequent paper.

\section{Acknowledgments}

We are grateful to R. Micnas and T. Kostyrko for helpful discussion. This work has financial supports from the Committee for Scientific Research, projects 2 P03B 104 11, 2P03B05709. Thanks are also due to the Polish-French cooperation through KBN-French Embassy agreement.

\section{References}

[1] J. Ranninger, S. Robaszkiewicz, Physica $B$ 135, 468 (1985); S. Robaszkiewicz, R. Micnas, J. Ranninger, Phys. Rev. B 46, 180 (1987).

[2] R. Micnas, J. Ranninger, S. Robaszkiewicz, Rev. Mod. Phys. 62, 113 (1990).

[3] J. Ranninger, J.M. Robin, Physica C 253, 279 (1995).

[4] G. Pawłowski, S. Robaszkiewicz, Mol. Phys. Rep. 12, 191 (1995); idem, Physica C, in press.

[5] S. Robaszkiewicz, G. Pawłowski, Physica C 210, 61 (1993). 